\title{
Sensing and control in dual-recycling laser interferometer gravitational-wave detectors
}

\author{
Kenneth A. Strain, Guido Müller, Tom Delker, David H. Reitze, David B. Tanner, \\ James E. Mason, Phil A. Willems, Daniel A. Shaddock, Malcolm B. Gray, \\ Conor Mow-Lowry, and David E. McClelland
}

\begin{abstract}
We introduce length-sensing and control schemes for the dual-recycled cavity-enhanced Michelson interferometer configuration proposed for the Advanced Laser Interferometer Gravitational Wave Observatory (LIGO). We discuss the principles of this scheme and show methods that allow sensing and control signals to be derived. Experimental verification was carried out in three benchtop experiments that are introduced. We present the implications of the results from these experiments for Advanced LIGO and other future interferometric gravitational-wave detectors. () 2003 Optical Society of America OCIS codes: $120.3180,120.5050,120.2230,350.1270$.
\end{abstract}

\section{Introduction}

Interferometric gravitational-wave observatories are expected to detect gravitational waves for the first time in the near future. These observatories will confirm the existence of an essential missing part of Einstein's general theory of relativity by directly observing gravitational waves. They will also, and with potentially much greater scientific yield, open a completely new branch of observational astronomy. Detectable gravitational waves are generated when objects with masses of the order of 1 solar mass undergo extreme acceleration. Most of the potential sources such as supernovas, black hole binaries, and neutron star mergers are among the most violent processes in the universe.

The first generation of laser interferometric

K. A. Strain (k.strain@physics.gla.ac.uk) is with the Institute for Gravitational Research, University of Glasgow, Glasgow G12 8QQ, Scotland. G. Müller, T. Delker, D. H. Reitze, and D. B. Tanner are with the Department of Physics, University of Florida, Gainesville, Florida 32611-8440. When this research was performed, J. E. Mason and P. A. Willems were with the LIGO Project, California Institute of Technology, Pasadena, California 91125. J. E. Mason is now with Lockheed Martin Advanced Technology Center, B201 L9-23, 3251 Hanover Street, Palo Alto, California 94304. D. A. Shaddock, M. B. Gray, C. Mow-Lowry, and D. E. McClelland are with the Department of Physics, Faculty of Science, Australian National University, Australian Capital Territory 0200, Australia.

Received 5 July 2002; revised manuscript received 14 November 2002.

0003-6935/03/071244-13\$15.00/0

(C) 2003 Optical Society of America gravitational-wave detectors [Laser Interferometer Gravitational Wave Observatory (LIGO ${ }^{1}$ ), VIRGO, ${ }^{2}$ GEO $600,{ }^{3}$ and TAMA ${ }^{4}$ ] are all either at advanced stages of construction or initial operation. These detectors are expected to be sensitive enough to open the field of gravitational-wave observation and are beginning substantial observing runs, operating in coincidence. All these systems employ detectors based on Michelson interferometers to measure tiny oscillating strains in space associated with gravitational radiation. It is likely that more-sensitive detectors are needed to give a reasonable rate of detections of a range of signal types.

In general the detector operates by transducing the optical phase change produced by a passing gravitational wave into an amplitude change measurable at a photodetector. In this paper we describe the principles of length-sensing and control schemes for an advanced interferometer configuration: the dualrecycled cavity-enhanced Michelson interferometer. It is the first of four papers, all published in this issue. The three others ${ }^{5-7}$ describe different realizations of length-sensing and control schemes.

The detectors used in the first-generation systems include various enhancements of the basic Michelson interferometer to improve sensitivity. The incorporation of Fabry-Perot cavities into arms of the LIGO, VIRGO, and TAMA interferometers allows the optical phase shift to be enhanced as, in effect, the light beams make multiple traversals of the varying optical path (in the original Michelson-Morley experiment folded arms were used to gain an increase in sensitivity $\left.{ }^{8}\right)$. Additional components are used to de- 
liver a sufficient photon flux to the beam splitter: a relatively powerful laser employed in combination with the technique of power recycling ${ }^{9,10}$ used in all first-generation systems. A related technique, signal recycling, ${ }^{11}$ provides control of the response of the interferometer. The combination of power recycling and signal recycling is conventionally termed dual recycling. Signal recycling is discussed in Subsection 1.A. Of the first-generation detectors, only GEO 600 employs signal recycling.

It is expected that in its initial configuration LIGO should detect up to a few events per year of operation. ${ }^{12}$ Improving the amplitude sensitivity by a factor of 10 increases the observation range for a given signal source strength by a factor of 10 and increases the event rate by approximately 3 orders of magnitude depending on the spatial distribution of the gravitational-wave sources (on length scales much larger than the mean separation of galaxies near our own). The potential for a huge leap in the scientific yield from the observatories provides sufficient motivation for rapid development of the necessary technology. We consider modifications of the optical configuration that should allow an order of magnitude improvement in sensitivity, then introduce a sensing and control scheme that should allow the interferometer to operate.

During normal operation the first-generation instruments will contain of the order of $0.1 \mathrm{~J}$ of light or $5 \times 10^{17}$ photons (in all cases from Nd:YAG lasers emitting light at $1064 \mathrm{~nm}$ ). The stored energy sets a performance limit, the shot-noise limit, over much of the important frequency range of the instruments from above approximately $100 \mathrm{~Hz}$ to an upper useful limit of several kilohertz. At lower frequencies (below roughly $100 \mathrm{~Hz}$, but varying among the different detectors) displacement noise of the mirror surfaces is dominant, arising from various sources including thermal noise and seismic noise. We do not discuss mirror displacement noise in this paper.

In LIGO the core of the detector is a power-recycled Fabry-Perot Michelson interferometer. This interferometer topology is shown in Fig. 1. The arms are $4 \mathrm{~km}$ long, and vacuum systems of this length have been built at both of the LIGO facilities. The most efficient operation of this type of interferometer requires the output port to be held close to a dark fringe (interference minimum). The Fabry-Perot arm cavities are chosen such that the width (in frequency) of their resonances is of the order of the desired signal bandwidth of the interferometer, i.e., approximately $100 \mathrm{~Hz}$. With 4-km-long arms this corresponds to a finesse of a few hundred; and with modern, low-loss optics, nearly all the light is reflected rather than being scattered or absorbed. With the interferometer held at a dark fringe, most of the light would be directed back out of the input port toward the laser. By adding a carefully chosen and positioned mirror, called the power-recycling mirror, one can optimize the circulating laser power and maximize the number of photons stored in the interferometer, available to interact with gravitational radiation. The fre-

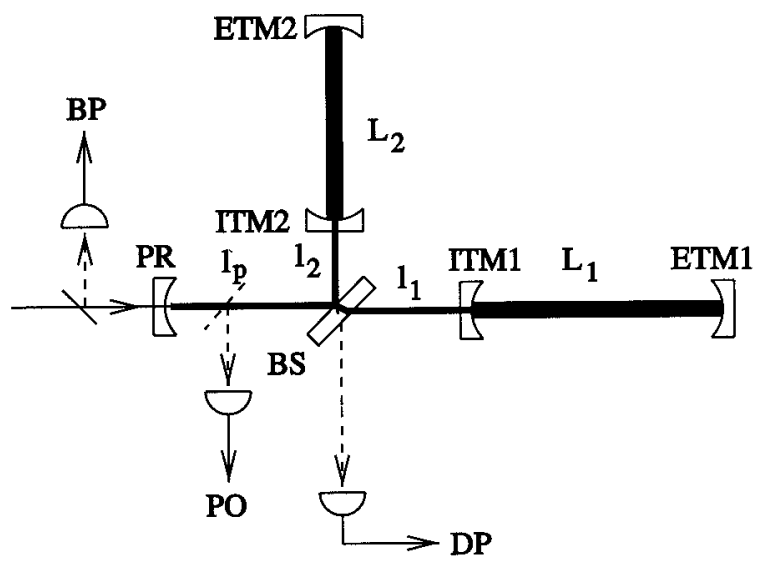

Fig. 1. Simplified diagram of an initial LIGO interferometer. Each interferometer consists of two arm cavities formed between the mirrors input test mass [ITM1(2)] and end test mass [ETM1(2)] of length $L_{1}$ and $L_{2}$ of approximately $4000 \mathrm{~m}$, whereas the other distances are of the order of a few meters. The beam splitter (BS) and power-recycling (PR) mirror are shown, the function of the latter is discussed in the text. The input light, from the injection optics, enters at the left, and the signal is detected at the dark port (DP) photodetector. Note that it is important to distinguish the macroscopic mirror spacings from the microscopic values that determine the phase of the light at refection from the optics. Microscopic displacements are described by their effect on the phase of the light fields, modulo one cycle. BP, bright port; DP, dark port; PO, pickoff.

quency response of such an interferometer therefore is determined almost entirely by the arm cavities: The response of the whole interferometer is dominated by the roll-off at the corner frequency of the individual cavities. A wider bandwidth with equally good peak sensitivity would provide a better chance of detecting gravitational waves. However, there is always a trade-off between bandwidth and peak sensitivity for a fixed stored light energy. Technical limitations on the stored energy have forced all firstgeneration detectors to adopt an interferometer bandwidth of the order of $100 \mathrm{~Hz}$. Such technical limitations and expectations of the likely gravitational-wave signal spectra would have been taken into account in the choice of the bandwidth for each detector.

The scientific aims and benefits of Advanced LIGO have been detailed elsewhere. ${ }^{13}$ In general terms the intention is to improve the peak gravitationalwave (strain) sensitivity by over 1 order of magnitude beyond that of the initial detectors and to allow observations at lower frequencies by an extension of the frequency range down to a lower cutoff of approximately $10 \mathrm{~Hz}$. Target sensitivity curves are shown in Fig. 2. This shows an estimate of the sensitivity that should be achievable given the application of technology currently under development within the LIGO Science Collaboration (LSC). The LIGO observatories were designed to accommodate upgraded detectors within the vacuum systems, clean rooms, and other facilities built to house the initial detectors. The first stage of upgrades is called Advanced LIGO 


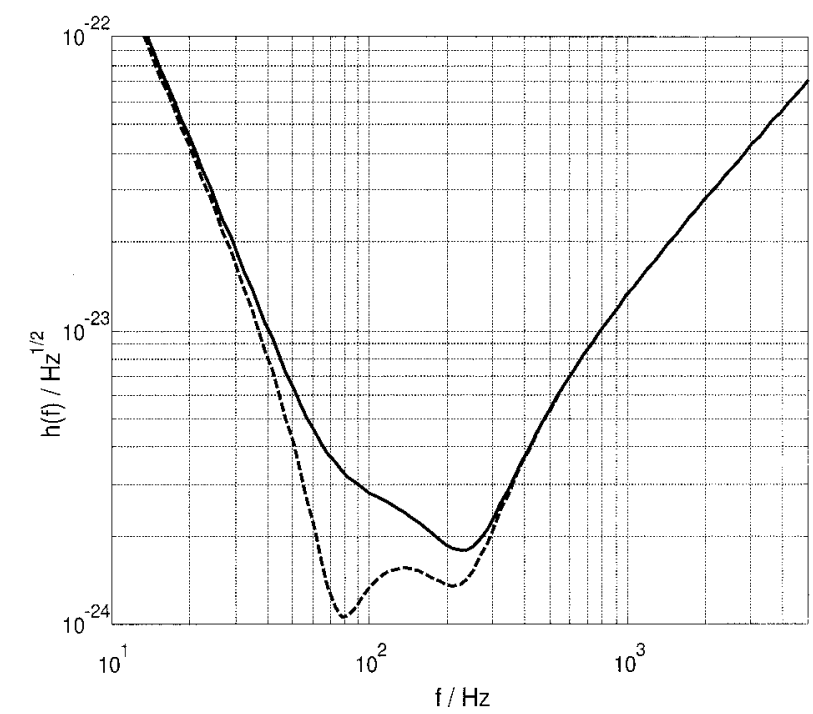

Fig. 2. Example sensitivity target for future instruments such as Advanced LIGO. The strain-noise amplitude spectral density is plotted over the frequency range of useful sensitivity. The solid curve shows an estimate of the total noise from all contributions, whereas the dashed curve shows just the noise associated with the optical sensing system. Over much of the frequency band shown, the proposed sensitivity is at least an order of magnitude better than that of first-generation instruments.

and is planned to consist of the replacement or enhancement of the interferometric detectors at both LIGO sites.

\section{A. Signal-Recycling Techniques for Advanced Interferometers}

The sensitivity of the optical system to gravitational radiation within a well-defined signal frequency band could be improved when the frequency response of the interferometer is matched to the expected signal spectrum. This can be achieved in practice by the application of signal recycling, a technique that requires the addition of one mirror, and a consequential increase in system complexity. Signal recycling removes the relationship between cavity bandwidth (or finesse) and interferometer response to an extent that is limited by loss in the optical system.

With the interferometer controlled to maintain the output port at an interference minimum, any small change to the interferometer that is common to the two arms will cause an effect at the input port, whereas any differential change, including a gravitational-wave signal, will appear at the output port. To understand the operation of the interferometer, it is easiest to consider that the effect of the gravitational radiation is to phase modulate the light in the arms of the interferometer. Then differential phase modulation sidebands will propagate to the output port where they can be detected. In a perfect interferometer, with a perfectly dark fringe (zero carrier field) at the output port, the sidebands could be measured by the addition of a small local oscillator component (with either homodyne or heterodyne detection).
Any common light field approaching the beam splitter from the arms interferes constructively in the direction toward the illuminating laser (the input or bright port). This light field does not contain any information about the gravitational waves, and so the power-recycling mirror placed at the input port does not affect the frequency response of the interferometer to gravitational waves. Instead it has the effect of enhancing the overall light energy stored in the interferometer.

Signal recycling ${ }^{10,14}$ operates by one adding coherently the phase modulation sidebands back into the interferometer. These then add with the sidebands produced at earlier and later times. The reflectivity of the signal-recycling mirror controls the effective number of round trips over which the sidebands are summed, and hence the bandwidth of the interferometer. The position of the signal-recycling mirror controls which sideband frequencies will add constructively and which will add destructively, thus determining the tuning of the interferometer. The signal-recycling mirror is partially transmissive, and the filtered light field can be detected as in the simpler system.

The frequency response of the interferometer is determined by the position of the signal-recycling mirror. Conventionally the special case of maximum response at zero signal frequency is termed tuned; all other cases, with peak response at a finite frequency, are called detuned. The detuning is usually described by either the frequency of peak response or by the shift of the signal-recycling mirror away from the tuned point-often in terms of an optical phase shift. We adopt this convention.

\section{B. Increasing the Stored Light Energy}

First-generation interferometers should operate at the photon-counting statistics (or shot-noise) limit of sensitivity. To provide an order of magnitude improvement in sensitivity it is necessary to increase the number of photons by approximately 2 orders of magnitude, or equivalently increase the stored energy to approximately $10 \mathrm{~J}$. This can be accomplished in part with each photon stored for a longer period of time, but for the most part must be achieved by an increase in the photon flux from the illuminating laser. The first-generation instruments use $\sim 10$-W lasers and store the photons for $1 \mathrm{~ms}$ on average. Advanced LIGO will use $\sim 180$-W lasers and achieve at least double the storage time through reduced optical loss, mainly in mirror coatings. This would provide an improvement in sensitivity by $\sim 5$ times, still less than is desired; the remaining factor of $\sim 2$ will be obtained by response shaping by use of signal recycling. In the discussion below it should be noted that, although the stored light energy determines the shot-noise-limited sensitivity, it is the circulating optical power that causes technical difficulties because of heating of the optics.

Although the shot noise can be decreased by an increase in the stored energy, another noise contribution, the quantum radiation pressure noise, in- 
creases. This produces a noise minimum that is called the standard quantum limit. It has recently been realized that, in signal-recycling interferometers, the uncertainty principle is more complicated than had been assumed, providing the opportunity to tune the interferometer parameters so that it can operate beyond the standard quantum limit. ${ }^{15}$ In fact the lower curve shown in Fig. 2 crosses below the standard quantum limit.

It is possible to fabricate mirrors with coating absorption coefficients of less than $10^{-6}$. A 4-km-long cavity fabricated from such mirrors can store $\gg 10$ $\mathrm{J}$ of light energy in a single optical mode. A problem arises, however, when one considers that, with normal transmissive optics, the light must pass through substrates of input test masses (ITMs) and the beam splitter (see Fig. 1) on the way to the cavities. Transmissive substrate materials considered for use in gravitational-wave detectors (fused silica and sapphire) cannot support circulating powers of more than a few kilowatts) without acquiring a strong thermal lens (absorption leads to localized heating, and so to refractive-index changes). ${ }^{16}$ Use of high-finesse arm cavities would allow the stored energy to be increased while reducing the power in the substrates, provided that the distortion that is due to heating of the coating is tolerable (this would require in practice either a coating loss of $\ll 1$ part per million, or otherwise some method of thermal compensation). In a power-recycled Michelson configuration, such highfinesse cavities would yield a frequency response too narrow to be of practical use in gravitational-wave detection. Signal recycling, however, can allow a useful response function to be obtained from a detector with high-finesse arm cavities. This was the motivation for the invention of the extension of signal recycling known as resonant sideband extraction. ${ }^{14}$ Resonant sideband extraction corresponds to strongly detuned signal recycling. Frequency responses of interferometers with high-finesse arm cavities, with and without resonant sideband extraction, are compared in Fig. 3. The curves shown were calculated according to the complete quantummechanical models of Ref. 15 and thus include all the effects of back-action of the light on the suspended optical components within the interferometer. It is this back-action that produces the poor sensitivity at Fourier frequencies much below $100 \mathrm{~Hz}$. In both cases the performance at low frequency could be improved by a reduction of the light energy stored in the interferometer, resulting, however, in poorer performance above $\sim 100 \mathrm{~Hz}$. In the signal-recycled case the detuning was selected to produce the minimum noise in the middle of the observation band. Optimization of the interferometer is nontrivial and we accomplished this here by using iterative numerical methods to give the best sensitivity to signals from the final stages of binary neutron star inspirals. In this example, the finesse of each arm cavity was 1250 , the signal-recycling mirror was $93 \%$ reflective, and the detuning phase of the signal recycling was 0.18 rad.

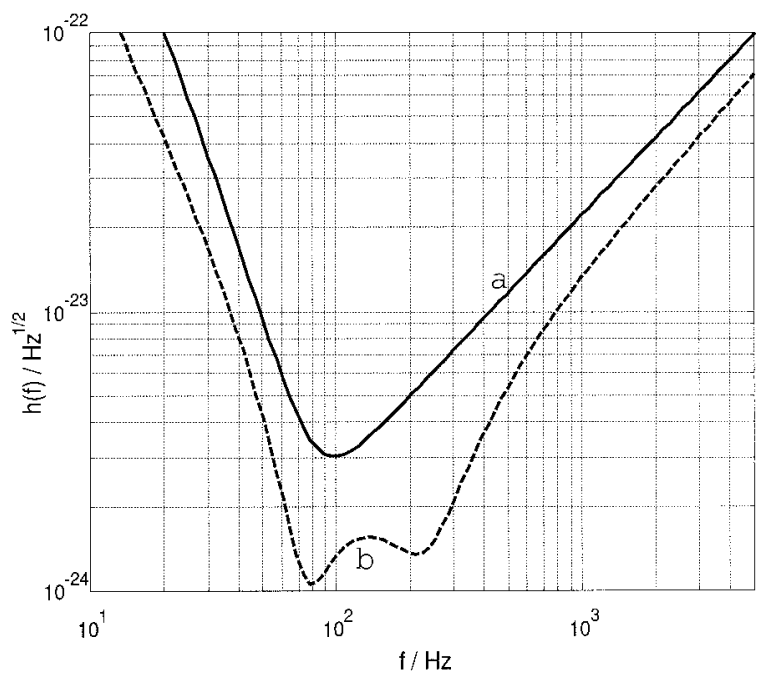

Fig. 3. Comparison of the sensitivity of power-recycling and dualrecycling interferometers. The solid curve shows the optical performance limit with power recycling only, whereas the dashed curve shows the result with the variant of dual recycling known as resonant sideband extraction. The parameters used are those proposed for Advanced LIGO (the most important of which are given in the text and are also those used in Fig. 2), except that the effect of signal recycling was suppressed in curve a.

In resonant sideband extraction, the signalrecycling mirror acts together with the ITMs to reduce the apparent finesse of the arm cavities for the gravitational-wave signal sidebands. The proposed interferometer configuration is shown in Fig. 4. The carrier light, being common mode and not reaching the output port, is not affected. In the same way that a lossless Fabry-Perot cavity with identical mirrors can have complete transmission at resonance, the signal-recycling mirror could, in a lossless interferometer, reduce the storage time in the arms to that of a single round trip. If the interference were perfect, no carrier light would reach the signal-recycling mirror, and the buildup of laser light power in the arms would not be affected. In a real detector, in the presence of loss and imperfect optical figure, there is a limit to how much the signal bandwidth can exceed that for the carrier, but a ratio of more than 1 order of magnitude can be realized. Thus the arm cavity finesse can be increased by a factor of 10 or more, thereby reducing the problems associated with the handling of increased light power, allowing a significant increase in stored energy.

\section{Methods of Sensing Interferometer Degrees of Freedom}

The mirrors forming the interferometer have to be aligned so that the correct optical modes are established; they must also be positioned to within a small fraction of a wavelength of the light to maintain the required resonant conditions (that is, to keep the arm cavities on resonance with the injected laser light, to maximize the effect of power recycling, to maintain the Michelson at the interference minimum, and to 


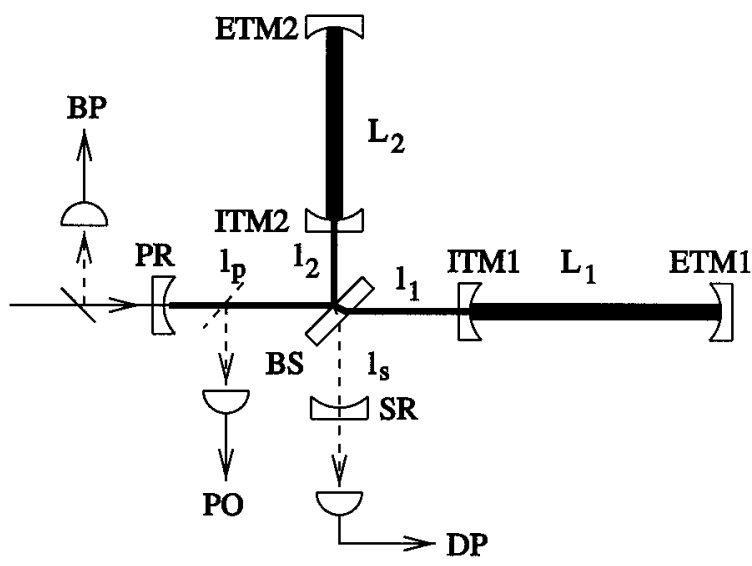

Fig. 4. Advanced LIGO interferometer consists of two arm cavities formed between the mirrors ITM1(2) and ETM1(2) of length $L_{1}$ and $L_{2}$. The distances of the arm cavities from the beam splitter (BS) are $l_{1}$ and $l_{2}$. The power-recycling (PR) mirror at a distance $l_{p}$ in front of the beam splitter and the signal-recycling (SR) mirror at $l_{\mathrm{s}}$ behind the beam splitter complete the interferometer. The lengths $L_{i}$ of the arm cavities are approximately $4000 \mathrm{~m}$ whereas the other distances are of the order of a few meters. These distances depend on the final length-sensing scheme and have to match the used modulation frequencies. Note that it is important to distinguish these macroscopic mirror spacings from the microscopic quantities that determine the phase of the light at refection from the optics. Microscopic displacements are described by their effect on the phase of the light fields. BP, bright port; DP, dark port; PO, pickoff.

select the correct frequency response provided by signal recycling).

The implementation of signal recycling increases the complexity of the sensing and control system required to hold all the components in the alignment and the position necessary to obtain the desired operation. In this paper we discuss the first stages in the research and development of the sensing and control systems of the Advanced LIGO interferometers.

To simplify the description of the control system we choose coordinates based on the spacings of mirrors that control these important aspects of the interferometer, rather than using coordinates associated with the individual mirrors. There are several possible basis sets, and the one presented here is chosen for simplicity of understanding. To this end we choose to take the beam splitter as a fixed reference and to give coordinates in terms of round-trip phase, neglecting complete cycles.

The gravitational-wave signal is detected through the differential optical path change it produces between the two long arms. The corresponding coordinate is called $\Phi_{-}$. Complementing this the coordinate reflecting the common-mode motion of the two arms is called $\Phi_{+}$. The position of the beam splitter, measured from the two nearest arm cavity mirrors, achieves the dark-fringe condition at the output port. However, it is convenient to take the beam splitter as fixed and to measure the positions of other nearby optics [ITMs and recycling mirrors
$(\mathrm{RMs})]$ with respect to it. In this picture the darkfringe condition is controlled by the relative spacing of the two ITMs from the beam splitter. This degree of freedom is called $\phi_{-}$. Again there is a corresponding common-mode degree of freedom from the two near-cavity mirrors to the power-recycling mirror: $\phi_{+}$. The last degree of freedom, along the axis of the optical mode of the interferometer, is that from the two near mirrors to the signal-recycling mirror: $\phi_{s}$. The power- and signal-recycling mirrors can be thought of as each forming a cavity with a complex mirror that is the rest of the interferometer. The two cavities are called the power-recycling cavity and signal-recycling cavity, respectively.

In addition to these are two corresponding angular degrees of freedom per linear degree of freedom. We do not discuss this aspect further in this paper.

It is necessary to measure (readout) a signal representing each of the named degrees of freedom. This can be done by a number of methods, but each is fundamentally an extension of the Pound-DreverHall technique. ${ }^{17}$ The general idea is to split the optical beam into several frequency components (normally by application of sinusoidal phase modulation at radio frequencies) such that in each region of the interferometer different combinations of the components are resonant. Signals extracted from the ports shown in Fig. 4 are then demodulated to measure the extent of beats between the components, revealing the resonant conditions and the relative positions of the mirrors.

It is difficult to design a sensing system that provides separate signals for each degree of freedom listed. However, it is acceptable from a control theory perspective that these signals be obtained in mixtures, provided that they can be separated cleanly after detection. The signals must be obtained in a linearly independent manner, and the mixing matrices should be robust against minor changes in interferometer parameters (such as might occur when mirror loss increases with time). Signals from the three ports used in LIGO (reflected, transmitted, and pickoff; see also Subsection 2.A) are not sufficient to control Advanced LIGO unless at least two modulation frequencies are employed. It is nontrivial to design a sensing system that decouples the five length-sensing signals obtained at three detection ports as a result of demodulation at one of two modulation frequencies (or at the beat frequency between the modulation frequencies).

In some situations it is difficult to arrange for the open-loop sensing system to be sufficiently separable. In this case one must seek to design a controller that operates to improve the separation through use of gain hierarchy. If two signals are mixed in two ports with one dominant in both ports, separation could be problematic (relying on the cancellation of two large numbers to extract a small one). However, if the large signal can be suppressed to a large degree by means of closing a servo loop around that degree of freedom, the situation can be improved. The servo loop is unlikely to be affected by the presence of a 
small signal from the other degree of freedom, but can suppress the large signal by the loop gain-possibly several orders of magnitude. This technique is used in LIGO. ${ }^{18}$

The sensing and control system developed for LIGO $^{19}$ was already thought to be quite complicated. There was some concern that the added complexity of signal recycling would make control of the new system intractable. This motivated the initiation of a research and development program by members of the LSC and was generally coordinated by that collaboration. Within this program three benchtop experiments have been completed as a first phase of research and development.

Results from the three initial experiments are reported in the companion papers. ${ }^{5-7}$ It should be noted that these experiments were self-contained and largely self-motivated.

The initial objective was to show that the selected interferometer configuration could be operated with a range of operational parameters and that there would be at least one viable sensing and control system; in fact far more than this was achieved.

\section{A. Mathematical Basis for the Design of Length-Sensing Systems}

Interferometric length-sensing and control schemes are often based on phase modulation and demodulation techniques that were introduced above. All schemes described here and in the companion papers make extensive use of these techniques, and it is appropriate to review the way in which these work.

A phase-modulated laser field can be described by a pair of sidebands separated from the central frequency component (the carrier) by the modulation frequency $\Omega$ :

$$
\begin{aligned}
E_{\text {in }}= & E_{0} \exp \left[i\left(\omega_{0} t+m \sin \Omega t\right)\right] \\
\approx & E_{0}\left\{\exp \left(i \omega_{0} t\right)+\frac{m}{2} \exp \left[i\left(\omega_{0}+\Omega\right) t\right]\right. \\
& \left.-\frac{m}{2} \exp \left[i\left(\omega_{0}-\Omega\right) t\right]\right\}
\end{aligned}
$$

where $E_{0}$ is the amplitude and $\omega_{0}$ is the angular frequency of the original laser field. The amplitude of each sideband is proportional to the modulation index $m$ provided that it is sufficiently small. Larger modulation indices would also create higher harmonics at $\omega_{0} \pm N \times \Omega$.

The reflected, transmitted, or internal pickoff field (a field extracted from within the power-recycling cavity, such as by the pickoff shown in Fig. 4) in any interferometer can be described as a linear combination of these frequency components with amplitudes and phases modified by the frequency-dependent transfer function $T(\omega)$ of the interferometer. Thus, in general,

$$
\begin{aligned}
E_{\text {out }}= & E_{0}\left\{T_{0} \exp \left(i \omega_{0} t\right)+\frac{m}{2} T_{+} \exp \left[i\left(\omega_{0}+\Omega\right) t\right]\right. \\
& \left.-\frac{m}{2} T_{-} \exp \left[i\left(\omega_{0}-\Omega\right) t\right]\right\},
\end{aligned}
$$

with the transfer function evaluated at the three particular values

$$
T_{0}=T\left(\omega_{0}\right), \quad T_{+}=T\left(\omega_{0}+\Omega\right), \quad T_{-}=T\left(\omega_{0}-\Omega\right) .
$$

The photocurrent that such a field generates in a fast photodetector is proportional to

$$
\begin{aligned}
I \propto\left|E_{0}\right|^{2}+2 m\left|E_{0}\right|^{2}\left\{\mathscr{R}\left[T_{0}\left(T_{+} *-T_{-} *\right)\right] \cos (\Omega t)\right. \\
\left.\quad+\mathscr{F}\left[T_{0}\left(T_{+} *+T_{-} *\right)\right] \sin (\Omega t)\right\},
\end{aligned}
$$

where the asterisk is used to denote complex conjugation. This signal will be demodulated with an ac coupled electronic mixer followed by a low-pass filter to yield signal

$$
\begin{aligned}
S= & 2 \mathscr{R}\left[T_{0}\left(T_{+}{ }^{*}-T_{-}{ }^{*}\right)\right] \cos (\alpha) \\
& -2 \mathscr{F}\left[T_{0}\left(T_{+}{ }^{*}+T_{-}{ }^{*}\right)\right] \sin (\alpha),
\end{aligned}
$$

where $\alpha$ is the tunable demodulation phase. The first term, proportional to $\cos (\alpha)$, is usually called the quadrature $(Q)$ signal. It is especially sensitive to differences in the amplitudes of the sidebands. The second component proportional to $\sin (\alpha)$ is the inphase (I) signal. It is primarily sensitive to phase changes of the carrier relative to the sidebands. Both parts of the signal vanish only if the carrier or both of the sidebands vanish, or if the transfer function of the carrier is real and the transfer functions of the sidebands are complex conjugated to each other (modulus over all phase in all three transfer functions). If this is not the case, as for example in a detuned configuration, the intensity oscillates with the modulation frequency, and only one specific demodulation phase causes the signal to vanish. This is similar to a simple heterodyne interferometer in which a single additional frequency component is added to the carrier:

$$
E_{\text {in }}=\left[E_{1}+E_{2} \exp (i \Omega t)\right] \exp \left(i \omega_{0} t\right) .
$$

The fields at a photodetector still depend on the frequency-dependent transfer function $T(\omega)$. The photocurrent can then be written as

$$
I=\left|T_{1} E_{1}\right|^{2}+\left|T_{2} E_{2}\right|^{2}+2\left|T_{1} E_{1}\right|\left|T_{2} E_{2}\right| \cos (\Omega t+\varphi),
$$

where $\varphi=\left[\arg \left(T_{1}\right)-\arg \left(T_{2}\right)\right]$ is the phase difference between the two transfer functions. Demodulation with $\cos (\Omega t+\psi)$ yields

$$
S=2\left|T_{1} E_{1} \| T_{2} E_{2}\right| \cos (\varphi-\psi) .
$$


Table 1. Five Relevant Longitudinal Degrees of Freedom in an Advanced LIGO Interferometer ${ }^{\alpha}$

\begin{tabular}{lcl}
\hline \multicolumn{1}{c}{ Description } & Symbol & Physical Distance \\
\hline Differential arm cavity & $\Phi_{-}$ & $2 k\left(L_{1}-L_{2}\right)$ \\
Common arm cavity & $\Phi_{+}$ & $2 k\left(L_{1}+L_{2}\right)$ \\
Differential Michelson & $\phi_{-}$ & $k\left(l_{1}-l_{2}\right)$ \\
Power-recycling cavity & $\phi_{+}$ & $k\left(2 l_{p}+l_{1}+l_{2}\right)$ \\
Signal-recycling cavity & $\phi_{s}$ & $k\left(2 l_{s}+l_{1}+l_{2}\right)$ \\
\hline
\end{tabular}

${ }^{a}$ It is convenient to describe the two arm cavities by use of their average or common length and their length difference or differential length instead of the individual lengths. The $k=2 \pi / \lambda$ is the wave number. Note that the phases given under the symbol column correspond to microscopic changes of the macroscopic lengths given in the physical distance column.

This signal disappears if either of the transfer functions is zero or if the demodulation phase is equal to $\varphi \pm \pi / 2$.

\section{B. Application to Signal-Recycling Interferometers}

The basic layout of the Advanced LIGO interferometer is shown in Fig. 4. The phase-modulated laser field enters the interferometer through the powerrecycling mirror. The beam splitter sends the beam toward the two arm cavities formed between the input test masses ITMs and the end test masses (ETMs). The recombined beam is then either sent back to the power-recycling mirror or to the signalrecycling mirror.

The operation of the gravitational-wave detector requires that the interferometer be held at the following working points:

1. The carrier has to be resonant in the arm cavities.

2. The carrier has to build up in the powerrecycling cavity and the arm cavities. Thus the arms must not only be on resonance with the carrier, but also have negligible loss.

3. The transmissivity of the carrier from the input port to the output port in the cavity-enhanced Michelson interferometer should be, at a minimum, limited only by asymmetry of the loss in the arms.

4. The tuning of the signal-recycling mirror depends on the targeted gravitational-wave frequency. The proposed mode of operation requires a particular positioning (or detuning) of the signal-recycling mirror to generate the response shown in Fig. 2.

The five longitudinal degrees of freedom are defined in more detail in Table 1. All five degrees of freedom have to be sensed and controlled by use of the signals taken at three locations as shown in Fig. 5. The bright port and dark port receive, respectively, all the light reflected and transmitted. The pickoff receives a small fraction of the light field inside the power-recycling cavity. This fraction is intended to be sufficiently small that its extraction does not significantly change the remaining internal fields. (The pickoff beam would normally be generated with the unavoidable reflection at an antireflec-

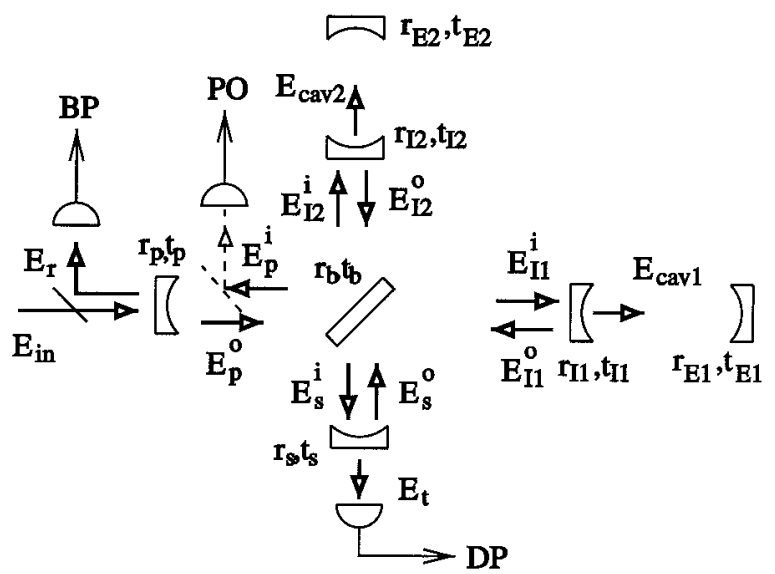

Fig. 5. Fields at the different locations in the interferometer. $E_{r}$ is the field that is reflected at the power-recycling mirror outside of the interferometer. It will be detected at the bright port (BP). $E_{t}$ is the field that is transmitted through the whole interferometer. It will be detected at the dark port (DP). The subscript $n$ of all the other fields $E_{n}{ }^{m}$ denotes the mirror at which the field is calculated. The superscript $m$ denotes the direction of the field. The pickoff (PO) field is proportional to $E_{p}{ }^{o}$.

tion coating, e.g., from the rear surface of the beam splitter.) The sideband frequencies $\Omega_{i}$ and the macroscopic distances are parameters that we can tune to find and optimize the five signals.

The transfer functions [see Eqs. (3)] for the carrier and each sideband are required to enable the calculation of the error signals. These transfer functions can be calculated from the following coupled set of equations that express the propagation of fields in the interferometer:

$$
\begin{aligned}
E_{r}= & t_{p} E_{p}{ }^{i}+r_{p} E_{\mathrm{in}}, \\
E_{t}= & t_{s} E_{s}{ }^{i}, \\
E_{p}{ }^{o}= & -r_{p} E_{p}{ }^{i}+t_{p} E_{\mathrm{in}}, \\
E_{p}{ }^{i}= & r_{b} E_{I 2}{ }^{o} \exp \left[-i k\left(l_{p}+l_{2}\right)\right]+t_{b} E_{I 1}{ }^{o} \\
& \times \exp \left[-i k\left(l_{p}+l_{1}\right)\right], \\
E_{s}^{o}= & r_{s} E_{s}^{i}, \\
E_{s}^{i}= & -r_{b} E_{I 1}^{o} \exp \left[-i k\left(l_{s}+l_{1}\right)\right]+t_{b} E_{I 2}^{o} \\
& \times \exp \left[-i k\left(l_{s}+l_{2}\right)\right], \\
E_{I 1}{ }^{i}= & -r_{b} E_{s}^{o} \exp \left[-i k\left(l_{s}+l_{1}\right)\right]+t_{b} E_{p}^{o} \\
& \times \exp \left[-i k\left(l_{p}+l_{1}\right)\right], \\
E_{I 1}{ }^{o}= & r_{\text {cav1 }}(k) E_{I 1}{ }^{i}, \\
E_{I 2}{ }^{i}= & r_{b} E_{p}^{o} \exp \left[-i k\left(l_{p}+l_{2}\right)\right]+t_{b} E_{s}^{o} \\
& \times \exp \left[-i k\left(l_{s}+l_{2}\right)\right], \\
E_{I 2}{ }^{o}= & r_{\text {cav2 }}(k) E_{I 2}{ }^{i} .
\end{aligned}
$$

Field $E_{\text {in }}$ is the component of the incoming laser field at angular frequency $\omega=c k$. The quantity $E_{r}$ is the field that is reflected at the power-recycling mirror outside of the interferometer; it will be detected at the bright port. Field $E_{t}$ is transmitted through the 
whole interferometer; it will be detected at the dark port. The subscript $n$ of all the other fields $E_{n}{ }^{m}$ denotes the mirror at which the field is calculated, where $p$ represents the power-recycling mirror, $s$ is the signal-recycling mirror, I1 is ITM1, and I2 is field $E_{t}$ at the dark port, and the pickoff field $E_{p}{ }^{o}$ are the fields used to generate the necessary sensing signals. After some algebraic manipulation, the transfer functions for these three fields are obtained as the following set of equations:

$$
\begin{aligned}
T_{r}(k) & =\frac{r_{p}\left[1-r_{s} \rho_{s} \exp \left(-i 2 k l_{s}\right)\right]+\left(r_{p}^{2}+t_{p}^{2}\right) \exp \left(-i 2 k l_{p}\right)\left[\rho_{p}+r_{s} X_{M} \exp \left(-i 2 k l_{s}\right)\right]}{1-r_{s} \rho_{s} \exp \left(-i 2 k l_{s}\right)+r_{p} \rho_{p} \exp \left(-i 2 k l_{p}\right)+r_{s} r_{p} X_{M} \exp \left[-i 2 k\left(l_{s}+l_{p}\right)\right]}, \\
T_{t}(k) & =\frac{t_{s} t_{p} \tau \exp \left[-i k\left(l_{p}+l_{s}\right)\right]}{1-r_{s} \rho_{s} \exp \left(-i 2 k l_{s}\right)+r_{p} \rho_{p} \exp \left(-i 2 k l_{p}\right)+r_{p} r_{s} X_{M} \exp \left[-i 2 k\left(l_{s}+l_{p}\right)\right]}, \\
T_{p}{ }^{o}(k) & =\frac{t_{p}\left[1-r_{s} \rho_{s} \exp \left(-i 2 k l_{s}\right)\right]}{1-r_{s} \rho_{s} \exp \left(-i 2 k l_{s}\right)+r_{p} \rho_{p} \exp \left(-i 2 k l_{p}\right)+r_{p} r_{s} X_{M} \exp \left[-i 2 k\left(l_{s}+l_{p}\right)\right]}
\end{aligned}
$$

ITM2. The superscript $m$ denotes the direction of the field traveling away $o$ (for out) or toward $i$ (for into) the mirror. The subscripts for the amplitude reflectivity $r_{n}$ and transmissivity $t_{n}$ (both real and positive) specify the optic: $b$ is the beam splitter, and $r_{\text {cav1 } 1}(k)$ and $r_{\text {cav2 } 2}(k)$ are the frequency-dependent reflectivity of each of the arm cavities given by

$$
\begin{aligned}
r_{\text {cav1 }(2)} & =\left[1-a_{1(2)}\right] \\
& \times \frac{+r_{I 1(2)}-\left[1-A_{I 1(2)}\right]\left[1-\frac{A_{E 1(2)}}{2}\right] \exp \left[-i 2 k L_{1(2)}\right]}{1-r_{I 1(2)}\left[1-\frac{A_{E 1(2)}}{2}\right] \exp \left[-i 2 k L_{1(2)}\right]} .
\end{aligned}
$$

Here the parameter $a_{1(2)}$ represents the loss in each of the short Michelson interferometer arms caused by the bulk absorption in the substrates-the ITMs and the beam splitter. (We note that this is not strictly correct as the loss in the beam-splitter substrate should appear also in one or the other of the recycling cavities. In a low-loss system, however, this simplification has a negligible effect on the result.) Furthermore, $A_{I 1(2)}$ and $A_{E 1(2)}$ represent the loss at the ITM and ETM surfaces and are given by

$$
A_{I}=1-r_{I}^{2}-t_{I}^{2} \ll 1, \quad A_{E}=1-r_{E}^{2} \ll 1 .
$$

The transmissivity of the ETM is included in the assessment of its loss.

The fields in each arm cavity can be calculated from the field impinging on each of the ITMs and are given by

$$
E_{\text {cav1(2) }}=\frac{t_{I 1(2)} E_{I 1(2)}^{i}}{1-r_{I 1(2)} r_{E 1(2)} \exp \left[-i 2 k L_{1(2)}\right]} .
$$

With the above quantities defined, the coupled set of Eqs. (9) can be solved to yield any field in the interferometer in terms of $E_{\text {in }}$. The resulting fields at each location will then reveal the transfer functions for an arbitrary frequency component in the input field to that location $T_{k}(k)=E_{k} / E_{\text {in }}$. The reflected field $E_{r}$ at the bright port, the transmitted
The contractions used in Eqs. (12) describe essentially the condition of the Michelson interferometer and are given by

$$
\begin{aligned}
\rho_{p}(k)= & \exp (-i k \bar{l})\left[\left(r_{b}{ }^{2} r_{\mathrm{cav} 2}+t_{b}{ }^{2} r_{\mathrm{cav} 1}\right) \cos \left(k l_{-}\right)\right. \\
& \left.+i\left(r_{b}{ }^{2} r_{\mathrm{cav} 2}-t_{b}{ }^{2} r_{\mathrm{cav} 1}\right) \sin \left(k l_{-}\right)\right], \\
\rho_{s}(k)= & \exp (-i k \bar{l})\left[\left(r_{b}{ }^{2} r_{\mathrm{cav} 1}+t_{b}{ }^{2} r_{\mathrm{cav} 2}\right) \cos \left(k l_{-}\right)\right. \\
& \left.-i\left(r_{b}{ }^{2} r_{\mathrm{cav} 1}-t_{b}{ }^{2} r_{\mathrm{cav} 2}\right) \sin \left(k l_{-}\right)\right], \\
\tau(k)= & \exp (-i k \bar{l}) t_{b} r_{b} \\
\quad \times[ & \left.\left(r_{\mathrm{cav} 1}-r_{\mathrm{cav} 2}\right) \cos \left(k l_{-}\right)-i\left(r_{\mathrm{cav} 1}+r_{\mathrm{cav} 2}\right) \sin \left(k l_{-}\right)\right],
\end{aligned}
$$

$X_{M}(k)=\tau^{2}(k)-\rho_{p}(k) \rho_{s}(k)$,

where, in turn, $\tau(k)$ is introduced to represent the transmissivity of the cavity-enhanced Michelson interferometer (from the bright port to the dark port and vice versa). Also $\rho_{p}(k)$ and $\rho_{s}(k)$ are its reflectivity as seen from the power- and signal-recycling mirrors, respectively. The quantities $\bar{l}=l_{1}+l_{2}$ and $l_{-}=l_{1}-l_{2}$ are the sum and the difference of the short Michelson interferometer arms (note that $l_{+}$includes the path to the power-recycling mirror as distinct from $\bar{l}$ that does not).

Equations (13)-(16) express the general solution for the fields in the interferometer. They can be best handled numerically or by computer symbolic algebra. To obtain a practical understanding of the design of a sensing system, however, it is beneficial to obtain a solution linearized about the desired operating point, as set out below.

A small detuning of $\delta L_{i}$ of the length of arm cavity $i$ will result in a small phase shift in the reflectivity of the arm cavities:

$r_{\mathrm{cav} 1(2)}\left[\Phi_{1(2)}\right] \approx-1+a_{1(2)}+\frac{2 A_{1(2)}}{T_{I 1(2)}}+i \Phi_{1(2)} \frac{4}{T_{I 1(2)}}$,

where $\Phi_{i}=2 k_{c} \delta L_{i} \ll 1$ is the round-trip phase shift of the field. In approximation (17) we used the fact that the reflectivity of the end mirror is close to unity 
compared with the reflectivity of the input mirror $\left(r_{I} r_{E} \approx r_{I}\right)$. Also

$$
T_{1(2)}=t_{1(2)}^{2} \equiv T_{I}
$$

is the intensity transmissivity of the input mirror and

$$
A_{1(2)} \equiv A_{I 1(2)}+\frac{\left(1+r_{I}\right)}{2} A_{E 1(2)}
$$

represents the loss in the cavity because of the imperfection of both mirrors.

If the Michelson interferometer is close to its locking point for the carrier, the argument in the trigonometric functions in Eqs. (13), (14), and (15) can be replaced, noting that

$$
k_{c} l_{-}=N 2 \pi+\phi_{-},
$$

where $k_{c}$ is the wave number for the carrier; we also have

$$
\begin{aligned}
& \cos \left(k_{c} l_{-}\right) \approx 1, \\
& \sin \left(k_{c} l_{-}\right) \approx \phi_{-} .
\end{aligned}
$$

The reflectivity and transmissivity of the Michelson interferometer are then given by

$$
\begin{aligned}
\rho_{p}\left(k_{c}\right) & \approx-\exp \left(-i k_{c} \bar{l}\right)\left[1-\bar{a}-\frac{2 \bar{A}}{T_{I}}-i \frac{2 \Phi_{+}}{T_{I}}\right] \approx \rho_{s}\left(k_{c}\right), \\
\tau\left(k_{c}\right) & \approx \exp \left(-i k_{c} \bar{l}\right)\left[i\left(\phi_{-}+\frac{2 \Phi_{-}}{T_{I}}\right)+\frac{2 \Delta A}{T_{I}}+\Delta a\right], \\
X_{M} & =-\exp \left(-i 2 k_{c} \bar{l}\right)\left(1-2 \bar{a}-\frac{4 \bar{A}}{T_{I}}-i \frac{4 \Phi_{+}}{T_{I}}\right) .
\end{aligned}
$$

Here we replaced the losses and phase shifts of the individual arms and arm cavities by their differential and common values. We also assumed that the beam splitter has exactly 50:50 reflectivity and transmittance. The substitutions are defined by the following set of six expressions:

$$
\begin{gathered}
\bar{a}=\frac{a_{1}+a_{2}}{2}, \\
\Delta a=\frac{a_{1}-a_{2}}{2}, \\
\bar{A}=\frac{A_{1}+A_{2}}{2}, \\
\Delta A=\frac{A_{1}-A_{2}}{2}, \\
\Phi_{+}=\Phi_{1}+\Phi_{2}, \\
\Phi_{-}=\Phi_{1}-\Phi_{2} .
\end{gathered}
$$

Substituting the above approximations [(23)-(31)] into the set of Eqs. (12) yields the final linearized result:

$$
\begin{aligned}
T_{r}\left(k_{c}\right) & =T_{r}^{0}\left(k_{c}\right)+i\left(T_{I} \phi_{+}+2 \Phi_{+}\right) t_{r}^{0}\left(k_{c}\right) \\
T_{r}^{0}\left(k_{c}\right) & \equiv \frac{-T_{p} T_{I}+2 T_{I} \bar{a}+4 \bar{A}}{T_{p} T_{I}+2 T_{I} \bar{a}+4 \bar{A}} \\
t_{r}^{0}\left(k_{c}\right) & \equiv \frac{4 T_{p} T_{I}}{\left(T_{I} T_{p}+2 T_{I} \bar{a}+4 \bar{A}\right)^{2}}, \\
T_{t}\left(k_{c}\right) & \approx t_{t}^{0}\left(k_{c}\right)\left[i\left(T_{I} \phi_{-}+2 \Phi+\right)+\Delta A+T_{I} \Delta a\right] \\
t_{t}^{0}\left(k_{c}\right) & \equiv \frac{4 t_{s} t_{p} T_{I}}{\left(T_{I} T_{s}+2 T_{I} \bar{a}+4 \bar{A}\right)\left(T_{I} T_{s}+2 T_{I} \bar{a}+4 \bar{A}\right)}, \\
T_{p}\left(k_{c}\right) & \approx T_{p}^{0}\left(k_{c}\right)-i\left(T_{I} \phi_{+}+2 \Phi_{+}\right) t_{p}^{0}\left(k_{c}\right) \\
T_{p}{ }^{0}\left(k_{c}\right) & \equiv \frac{2 t_{p} T_{I}}{T_{I} T_{p}+2 T_{I} \bar{a}+4 \bar{A}}, \\
t_{p}^{0}\left(k_{c}\right) & \equiv \frac{4 t_{p} T_{I}}{\left(T_{p} T_{I}+2 T_{I} \bar{a}+4 \bar{A}\right)^{2}} .
\end{aligned}
$$

In these expressions we also used the fact that the round-trip losses in the arm cavities are much smaller than the transmission of the input mirror, i.e.,

$$
\frac{\bar{A}}{T_{I}}, \quad \frac{\Delta A}{T_{I}} \ll 1,
$$

and that the carrier is on resonance in both recycling cavities so that

$$
\begin{aligned}
\exp \left[-i k_{c}\left(2 l_{p}+\bar{l}\right)\right] & \approx 1+i \phi_{+}, \quad \exp \left[-i k_{c}\left(2 l_{s}+\bar{l}\right)\right] \\
& \approx-1-i \phi_{s} .
\end{aligned}
$$

The second half of Approximations (41) is valid only for signal recycling where the carrier would be resonant in the signal-recycling cavity, but to calculate the equivalent for our required case of detuned dual recycling (or resonant sideband extraction) we only have to make the substitutions

$$
r_{s} \rightarrow r_{s} \exp \left(-i \phi_{s}\right), \quad t_{s} \rightarrow t_{s} \exp \left(-i \phi_{s} / 2\right)
$$

in the transfer function $T_{t}\left(k_{c}\right)$. The quantities $T_{r}\left(k_{c}\right)$ and $T_{p}\left(k_{c}\right)$ do not change.

Inspection of Eq. (32) and approximations (35) and (37) yields a great deal of information about the nature of any practical length-sensing scheme. In particular, the Michelson interferometer separates the common $\left(\phi_{+}, \Phi_{+}\right)$from the differential $\left(\phi_{-}, \Phi_{-}\right)$degrees of freedom, but not the common or differential degrees of freedom from themselves. It also does not provide any control signal that is first-order dependent on $\phi_{s}$. In addition, the transfer functions depend more strongly on the cavity degrees of freedom 
$\left(\Phi_{-}, \Phi_{+}\right)$than on the auxiliary common and differential degrees of freedom $\left(\phi_{-}, \phi_{+}\right)$. Separating the mixed signals of widely varying magnitudes is the primary challenge to the designers of a sensing scheme.

The gravitational-wave signal should be detected with the highest possible sensitivity. Therefore the carrier transmitted to the dark port will be used to sense $\Phi_{-}$. The carrier should also be used to detect $\Phi_{+}$. The role of the additional frequency components injected into the system is to provide us with the necessary local oscillators at all detection ports and to generate additional control signals for the auxiliary degrees of freedom $\left(\phi_{-}, \phi_{+}, \phi_{s}\right)$. The three companion papers describe in detail three different solutions to this problem.

\section{Sensing and Control in Advanced Interferometers}

An ideal sensing system would have five outputs each corresponding to one of the sensed degrees of freedom. This is difficult (if not impossible) to achieve even in the region of parameter space around the desired operating point where the signals are linearly dependent on the sensed degrees of freedom [see the linearized Eq. (32) and approximations (35) and (37)]. It is noted that, provided the five signals contain linearly independent mixtures of the five degrees of freedom, a stable control system can be constructed. This suggests use of a sensing matrix representing the relationship between interferometer degrees of freedom and the five outputs. Ideally the matrix would be diagonal, but off-diagonal elements that are no larger than the diagonal elements are usually tolerable (with the obvious exceptions corresponding to linear dependence). It is noted that on-diagonal matrix elements vary by several orders of magnitude.

One of the main difficulties encountered by the designer of a sensing and control system for interferometers with high-finesse arm cavities is the overwhelming size of the signals representing the cavity degrees of freedom $\Phi_{-}$and $\Phi_{+}$in the measurement outputs designed to measure the other degrees of freedom. This is true wherever the beats between sidebands and the carrier are used to form the measurement signal (i.e., demodulation at a frequency equal to one of the modulation frequencies). During the progress of the benchtop experiments, it was realized that the technique of double demodulation could be used to reduce the significance of this problem. One must make a measurement that minimizes sensitivity to the strong carrier phase signals produced by the arm cavities. This can be done when a signal is derived from the beat between two sets of modulation sidebands, each chosen to be far off resonant in the arm cavities. Then one can realize that sequential demodulation at the two modulation frequencies is equivalent to demodulation at the beat frequency. (This is largely a matter of convenience as it avoids the need to synthesize a sufficiently stable beat-frequency local oscillator.) This technique makes the generation of nearly diagonal sensing matrices considerably simpler than it would be other- wise. The double-demodulation technique is at the heart of the sensing scheme proposed for Advanced LIGO.

\section{A. Results from the Benchtop Experiments}

Three benchtop experiments were carried out to explore a range of strategies for sensing and control in dual-recycling interferometers. The experiments were successful and yielded a rich set of results. Some key points are summarized here, but for full detail the reader is referred to the companion papers. ${ }^{5-7}$

The sensing scheme developed by Mason and Williams ${ }^{6}$ was proposed as a minimal extension to the methods used in the LIGO interferometers. ${ }^{19}$ The addition of a single sideband (rather than a pair of phase modulation sidebands) achieves the basic goal of providing five output signals from three detection ports. The scheme was successful, as detailed in the paper, where a number of technical limitations are also discussed. An interesting feature of this design was use of single sideband modulation (see also Subsection 3.B).

The scheme developed and tested by Müller et al. ${ }^{5}$ was a slightly more radical approach to the same problem, but with similar goals and results. Two sets of phase modulation sidebands were applied to the input light, and the signals were obtained by suitable demodulation of these, including double demodulation where appropriate. The modulation frequencies and macroscopic mirror spacings within the interferometer were chosen to meet the key aim of separating the signals for the auxiliary degrees of freedom as much as possible. This was achieved by the choice of one modulation frequency such that little of its power was transmitted to the dark port, whereas the other would be transmitted to the bright port as strongly as possible. The former sidebands would not show significant phase shift from the signal-recycling mirror but would be strongly influenced by the power-recycling cavity length, whereas the latter set of sidebands would be more equally affected by the signal and power cavities. In a detuned interferometer one of the pair of sidebands intended to be transmitted to the dark port would be transmitted more efficiently than the other. Müller et al. show how this scheme can be made to operate.

The research undertaken by Mason and Williams ${ }^{6}$ and Müller et al..$^{5}$ has demonstrated that there are a number of methods available to allow the interferometer degrees of freedom to be read out in a manner suitable for the control of the interferometer. Most significantly, new methods for obtaining clean signals for $\phi_{+}, \phi_{-}$, and $\phi_{s}$ have been proven. It has been shown too that the readout scheme for an interferometer with fixed signal-recycling tuning can be a relatively minor extension of existing methods.

Advanced LIGO, as currently proposed, is planned to operate with constant detuning of the signalrecycling cavity. However, it would be possible to operate a similar configuration in a more versatile manner, for example, to perform a rapid adjustment 
of an interferometer to obtain enhanced sensitivity over a narrowed bandwidth or to follow a signal progressing through the frequency band. ${ }^{20}$ The research carried out by Shaddock et al. ${ }^{7}$ had as its goal the design and construction of a tunable interferometer. As described in that paper the basic approach was the same as in the other experiments, but with the added feature of tunability. Shaddock et al. describe in detail how the problems associated with tuning over a wide range could be handled by careful choice of design parameters. The experiment was successful and shows that tunable interferometers remain an option for the future, at the expense of a slight increase in complexity.

The results presented here and in the three companion papers have been applied to the design of sensing and control schemes for three different interferometers. The methods were first applied to the Advanced LIGO baseline interferometer design. This design is the responsibility of the LIGO laboratory and is the topic of continuing research by several groups within the LSC. Its complete description is beyond the scope of the present paper, but the design principles are presented in Subsection 3.B as an example of how the present results are applied to a practical problem.

The methods have also been used to design two prototype test interferometers, each designed to probe aspects of the Advanced LIGO design in further detail and with more realistic parameters than the benchtop experiments; one of these is in Glasgow, and the other is at the California Institute of Technology. The entire program is described in Ref. 21.

\section{B. Baseline Sensing Method for Advanced LIGO}

The sensing and control system for Advanced LIGO must satisfy a number of requirements: It must bring the interferometer from a random starting condition to the desired operating point, it must feedback correction signals to the positions of the optical components to hold the interferometer at the operating point to within some specified accuracy, and it must give a low-noise electronic signal containing the gravitational-wave information.

This is an extremely complex problem, and here we restrict our discussion to a possible method of the sensing of the signals required to provide linear control around the operating point. We do this from the point of view of explaining the design process rather than presenting a description of the design. We explicitly avoid describing the method used to read out the gravitational-wave signal; optimizing this signal is the subject of research.

As noted above, for a range of practical considerations, the entire optical field that is used to illuminate the interferometer, complete with modulation sidebands, must be passed through an optical cavity. (The main reason for this is to provide filtering of geometric changes in the illuminating field that could couple to the gravitational-wave channel in the case of imperfect geometry of the interferometer. ${ }^{22}$ ) Such a cavity is conventionally termed a mode cleaner, and in the case of the LIGO facilities this is a suspendedmirror, triangular ring cavity of $\sim 33-\mathrm{m}$ round-trip length in vacuum. This cavity, when resonant for the optical carrier frequency, will transmit efficiently any modulation sidebands at multiples of its free spectral range, i.e., frequencies given by $N \times 9 \mathrm{MHz}$, where $N$ is a natural number. The sensing system therefore is restricted to using frequencies from this finite list. Considerations of modulator, photodetector, and associated electronic design constrain the maximum modulation frequency to $\sim 200 \mathrm{MHz}$.

In the light of success having been achieved in both benchtop experiments, it appeared that the modulation frequencies could either be chosen to have a small ratio (as in the Mason and Williams ${ }^{6}$ experiment) or a much larger ratio. It was believed that there was some risk associated with small ratios (e.g., 3) because nonlinearity in the radio-frequency electronics could then easily lead to cross contamination of the signals (harmonics of the lower frequency contaminating the higher one).

With the above considerations in mind, the approach taken was to have one sideband at a high modulation frequency transmitted to the dark port, with the other pair of sidebands (at a much lower frequency) resonant within the power-recycling cavity only. No sideband would be resonant in the arm cavities of the interferometer. It is noted that, for a detuned interferometer, the signal-recycling cavity cannot be equally resonant for both of a pair of phase modulation sidebands. Hence only one of the highfrequency sidebands can be fully resonant. Although we would apply phase modulation at the input to the interferometer, the resulting internal light field would end up effectively as single sideband modulation as was employed in the Mason and Williams experiment.

To obtain the largest possible sensing signals it is important to ensure that the modulation sidebands are resonant in the interferometer. Achieving the necessary resonant conditions for the sidebands requires that the mirror (and beam splitter) spacings are chosen correctly. This was accomplished in the design by use of iterative numerical calculation.

Probably the most significant parameter is $l_{-}$, as this determines the frequency-dependent coupling from the power-recycling cavity to the signalrecycling cavity within the interferometer. If $l_{-}=0$, the interferometer would be a white-light interferometer and there would be no such coupling. Schnupp ${ }^{23}$ first highlighted the significance of making $l_{-} \neq 0$ to transfer modulation sidebands from the input to the output of the interferometer. For $l_{-} \ll$ $c / f_{\mathrm{sb}}$, where $f_{\mathrm{sb}}$ is the frequency of the modulation sideband in question, the coupling is proportional to $l_{-}$. The approach taken therefore was to optimize the coupling of a high-frequency sideband (we chose $180 \mathrm{MHz}$ ) to the signal-recycling cavity by the choice of the correct value of $l_{-}$.

The choice of the second modulation frequency is now relatively simple: It should be as low as possible $(9 \mathrm{MHz})$ to minimize its coupling into the signal- 
recycling cavity. With this choice and with the $l_{s}$ and $l_{+}$distances chosen to bring the sidebands on resonance within their respective cavities, the last step of the design process is the selection of ports at which to measure the five signals.

The signals associated with the arm cavities $\Phi_{-}$ and $\Phi_{+}$are obtained in the same manner independent of the details of the particular scheme. The differential signal $\Phi_{-}$is obtained when the dark port photodetector signal is demodulated at the frequency of the dominant sideband(s) at that port, in this case $180 \mathrm{MHz}$, whereas the $\Phi_{+}$information is obtained when the bright port photocurrent is demodulated at $9 \mathrm{MHz}$. In each case we are measuring the phase between the carrier resonant in the arm cavities and sidebands that are not resonant in these cavities (the classic Pound-Drever-Hall method).

Extracting the other three signals, representing the auxiliary degrees of freedom, is more complex. However, all signals are obtained by double demodulation at 180 and $9 \mathrm{MHz}$ in turn. The signals for $\phi_{-}$and $\phi_{s}$ are strongly coupled. Double demodulation of the bright port and dark port signals was found to yield a pair of mixed signals that could be optimized in their independence by choice of the demodulation phases in the double-demodulation process. The final signal for $\phi_{+}$can be obtained by double demodulation of the outputs from the bright port and pickoff photodetectors.

\section{Conclusion}

Enhanced interferometer designs should extend the sensitivity of gravitational-wave detectors. Three benchtop experiments have explored a range of sensing and control systems appropriate for application to a dual-recycled Fabry-Perot Michelson interferometer. These experiments were successful, in that they each demonstrated correct interferometer control and signal extraction. Among them they showed that the interferometer could be controlled in a wide range of operating modes and in accordance with the basic technical requirements imposed on modulation frequencies and detection locations within the LIGO context. Beyond this the tests have shown that it is possible to obtain flexibility beyond that required for Advanced LIGO, but for possible applications in other advanced detectors, namely, tuning over a wide range of frequencies and still maintain interferometer lock. Such tuning has been proposed in the context of increasing the sensitivity to gravitational waves from coalescing compact objects. Finally, the tests have shown that the analytical approach presented in this paper has useful application in the design and understanding of the sensing and control systems for dual-recycling interferometers.

It is interesting to note that many ideas were exchanged throughout the progress of the three experiments and in the design of the sensing scheme suitable for Advanced LIGO. This led to significant improvements in all three experiments and to the rapid formulation of an initial design for the Advanced LIGO sensing system.
The authors acknowledge support of colleagues within the LSC and financial support for this research from the Particle Physics and Astronomy Research Council and the University of Glasgow in the UK, from the National Science Foundation (PHY0070854, PHY-9986274, PHY-9801158, PHY9700601, and PHY-9210038), and from the Australian Research Council.

\section{References and Note}

1. A. Abramovici, W. Althouse, R. Drever, Y. Gursel, S. Kawamura, F. Raab, D. Shoemaker, L. Sievers, R. Spero, K. Thorne, R. Vogt, R. Weiss, S. Whitcomb, and M. Zucker, "LIGO: The Laser Interferometer Gravitational-Wave Observatory," Science 256, 325-333 (1992).

2. C. Bradaschia, R. Del Fabbro, A. Di Virgilio, A. Giazotto, H. Kautzky, V. Montelatici, D. Passuello, A. Brillet, O. Cregut, P. Hello, C. N. Man, P. T. Manh, A. Marraud, D. Shoemaker, J. Y. Vinet, F. Barone, L. Di Fiore, L. Milano, G. Russo, J. M. Aguirregabiria, H. Bel, J. P. Duruisseau, G. Le Denmat, Ph. Tourrenc, M. Capozzi, M. Longo, M. Lops, I. Pinto, G. Rotoli, T. Damour, S. Bonazzola, J. A. Marck, Y. Gourghoulon, L. E. Holloway, F. Fuligni, V. Iafolla, and G. Natale, "The Virgo project: a wide band antenna for gravitational wave detection," Nucl. Instrum. Methods Phys. Res. A 289, 518-525 (1990).

3. K. Danzmann, H. Lück, A. Rüdiger, R. Schilling, M. Schrempel, W. Winkler, J. Hough, G. P. Newton, N. A. Robertson, H. Ward, A. M. Campbell, J. E. Logan, D. I. Robertson, K. A. Strain, J. R. J. Bennett, V. Kose, M. Kühne, B. F. Schutz, D. Nicholson, J. Shuttleworth, H. Welling, P. Aufmuth, R. Rinkleff, A. Tünnermann, and B. Willke, "GEO 600-a 600-m laser interferometric gravitational wave antenna," in First Edoardo Amaldi Conference on Gravitational Wave Experiments, E. Coccia, G. Pizella, and F. Ronga, eds. (World Scientific, Singapore, 1995), pp. 100-111.

4. K. Tsubono, "300-m laser interferometer gravitational wave detector (TAMA 300) in Japan," in First Edoardo Amaldi Conference on Gravitational Wave Experiments, E. Coccia, G. Pizella, and F. Ronga, eds. (World Scientific, Singapore, 1995), pp. 112-114.

5. G. Müller, T. Delker, D. B. Tanner, and D. Reitze, "Dualrecycled cavity-enhanced Michelson interferometer for gravitational-wave detection,” Appl. Opt. 42, 1257-1268 (2003).

6. J. E. Mason and P. A. Willems, "Signal extraction and optical design for an advanced gravitational-wave interferometer," Appl. Opt. 42, 1269-1282 (2003).

7. D. A. Shaddock, M. B. Gray, C. Mow-Lowry, and D. E. McClelland, "Power-recycled Michelson interferometer with resonant sideband extraction," Appl. Opt. 42, 1283-1295 (2003).

8. A. A. Michelson and E. W. Morley, "On the relative motion of the earth and the luminiferous ether," Am. J. Sci. 35, 333-345 (1887).

9. R. W. P. Drever, "Interferometric detectors for gravitational radiation," in Gravitational Radiation, N. Daruelle and T. Piran, eds. (North-Holland, Amsterdam, 1983), pp. 321-328.

10. The idea of power recycling was proposed simultaneously by $R$. Schilling, Max-Planck-Institut fuer Quantenoptik, Garching, Germany.

11. B. J. Meers, "Recycling in laser-interferometric gravitationalwave detectors," Phys. Rev. D 38, 2317-2326 (1988).

12. E. S. Phinney, "The rate of neutron star binary mergers in the universe: minimal predictions for gravity wave detectors," Astrophys. J. Lett. 380, L17-L21 (1991).

13. C. Cutler and K. S. Thorne, "An overview of gravitational-wave sources," in Proceedings of the GR16 Conference on General 
Relativity and Gravitation, N. Bishop, ed. (World Scientific, Singapore, 2002).

14. J. Mizuno, K. A. Strain, P. G. Nelson, J. M. Chen, R. Schilling, A. Rüdiger, W. Winkler, and K. Danzmann, "Resonant sideband extraction: a new configuration for interferometric gravitational-wave detectors,” Phys. Lett. A 175, 273-276 (1993).

15. A. Buonanno and Y. Chen, "Quantum noise in second generation, signal-recycled laser interferometric gravitational-wave detectors," Phys. Rev. D 64, 77-98 (2001).

16. K. A. Strain, K. Danzmann, P. G. Nelson, A. Rüdiger, R. Schilling, and W. Winkler, "Thermal lensing in recycling interferometric gravitational wave detectors," Phys. Lett. A 194, 124132 (1994).

17. R. W. P. Drever, J. L. Hall, F. V. Kowalski, J. Hough, G. M. Ford, A. J. Munley, and H. Ward, "Laser phase and frequency stabilization using an optical resonator," Appl. Phys. B 31, 97-105 (1983).

18. M. Regehr, "Signal extraction and control for a interferometric gravitational wave detector," Ph.D. dissertation (California Institute of Technology, Pasadena, Calif., 1995).

19. P. Fritschel, R. Bork, G. González, N. Mavalvala, D. Ouimette, H. Rong, D. Sigg, and M. Zucker, "Readout and control of a power-recycle interferometric gravitational-wave antenna," Appl. Opt. 40, 4988-4998 (2001).

20. B. J. Meers, A. Krolak, and J. A. Lobo, "Dynamically tuned interferometers for the observation of gravitational waves from coalescing compact binaries," Phys. Rev. D 47, 21842197 (1993).

21. A. Weinstein, "Advanced LIGO optical configuration, prototyping, and modeling," Class. Quantum Grav. 19, 1575-1584 (2002).

22. A. Rüdiger, R. Schilling, L. Schnupp, W. Winkler, H. Billing, and K. Maischberger, "A mode selector to suppress fluctuations in laser beam geometry," Opt. Acta 28, 641-658 (1981).

23. L. Schnupp, "Internal modulation schemes," presented at the European Collaboration Meeting on Interferometric Detection of Gravitational Waves, Sorrento, Italy, 2 Oct. 1988. 